\title{
LOEWY SERIES OF CERTAIN INDECOMPOSABLE MODULES FOR FROBENIUS SUBGROUPS
}

\author{
ZONGZHU LIN
}

\begin{abstract}
We imitate some approaches in infinite dimensional representation theory of complex semisimple Lie algebras by using the truncated category method in the categories of modules for certain Frobenius subgroups of a semisimple algebraic group over an algebraically closed field of characteristic $p>0$. By studying the translation functors from $p$-singular weights to $p$-regular weights, we obtain some results on Loewy series of certain indecomposable modules.
\end{abstract}

\section{INTRODUCTION}

Lusztig's conjecture in the modular representation theory states that the character of a simple module can be calculated from a set of polynomials related with the affine Weyl group. Andersen's work [1] shows that Lusztig's conjecture is actually equivalent to the following fact: when one translates the simple module across an upper alcove wall, one will get a module which has Loewy length at most three. So the study of the translation functors turns out to be interesting and important. However, the behavior of translation functors is far from being understood. In this paper, we study the translation functors from $p$-singular weights to $p$-regular weights to obtain some results on the Loewy series of certain modules for Frobenius subgroups.

The representation theory of complex semisimple Lie algebras is an important source of methods in the representation theory of algebraic groups over fields of prime characteristic. These two theories are somehow parallel. The Lusztig conjecture is analogous to the Kazhdan-Lusztig conjecture and the Jantzen conjecture is analogous to the Jantzen conjecture for semisimple Lie algebras.

Irving has a series of papers $[9,10,12]$, in which he studied the translation functors in the infinite dimensional representation theory of semisimple Lie algebras, for which the Kazhdan-Lusztig conjecture has been proved. By studying the translation functors, he proves that the Verma modules and the projective covers of the simple modules with regular antidominant highest weights are rigid (i.e., the radical and socle filtrations coincide). Furthermore, their socle filtrations can be calculated from the Kazhdan-Lusztig polynomials. Andersen and Kaneda have imitated Irving's approach in the characteristic $p$ case. It turns out that Lusztig's conjecture gives not only the multiplicity of a simple module in a Weyl module, but also its position in the radical structure of that

Received by the editors January 22, 1990 and, in revised form, May 10, 1990.

1980 Mathematics Subject Classification (1985 Revision). Primary 20G05; Secondary 18G05. 
Weyl module (in the generic situation). So the situation is completely analogous to the case of the semisimple Lie algebra.

In order to make the idea clear, we recall some facts in the category $\mathscr{O}$ for a complex semisimple Lie algebra. The Verma modules with dominant highest weights are projective and the projective cover of a simple module with regular antidominant highest weight is achieved by the translation functor from the Verma module $M(-\rho)$. In the case of characteristic $p$, Koppinen in [16] has noticed that around a special point, the situation is similar to that of Verma modules. This idea also appears in the work of Andersen and Kaneda [2].

The main idea in this paper is to imitate Irving's method in studying the translation functors for semisimple Lie algebras. We need some categories which have the similar property as the category $\mathscr{O}$ does. These categories can be obtained by truncating the usual category. In these categories, the injectives are smaller, so homological methods can be applied to the injective modules to study the structure of some interesting modules such as those obtained from induced modules via translation functors from $p$-singular weights to $p$-regular weights.

In $\S 1$, we give the definition of the truncated category. In order to avoid the complications when a weight is close to a dominant chamber wall, we work over the Frobenius subgroups $G_{r} T$. There are two truncation functors, which are the left and right adjoint functors of the inclusion functor. They are right and left exact respectively and related by the duality functor introduced in [7].

In $\S 2$, we study the injective and projective modules in the truncated category. The structure of the projective modules can be obtained from that of injective modules via the duality functor. So we concentrate on the injective modules. We prove that the indecomposable injective modules have a "good" filtration and the so-called "Brauer-Humphreys" reciprocity holds. Then we imitate the approach of Andersen and Kaneda, and give an upper bound for the socle series of injective modules.

In $\S 3$, we study the translation functor in the truncated category and relate them to injective modules. Then using the theory we have established in truncated categories, we get some properties of the usual translation functors.

In $\S 4$, assuming the Lusztig conjecture, we prove that, in a $p$-singular block, the injective modules and the induced modules have the expected Loewy length, which has been proved by Andersen and Kaneda to be a lower bound. It is also proved that the module obtained via translating an induced module with $p$ singular highest weight to a $p$-regular weight is rigid and the upper bound given in $\S 2$ for these modules is the socle filtration. We expect the induced modules with $p$-singular weights to be rigid. Then the filtrations obtained from the socle and radical filtrations of induced modules with $p$-regular highest weights via translation functors are Loewy filtrations, hence the socle and radical filtrations of the induced modules with $p$-singular highest weights. This has been verified for rank 2 groups $[17,18]$.

Notations. $G$ is a connected and simply connected semisimple algebraic group defined over an algebraically closed field $k$ of characteristic $p>0$. We suppose that $G$ is defined on the prime field $\mathbf{F}_{p}$. Let $T$ be a split maximal torus of $G$ and $X(T)$ the character group of $T$. The elements of $X(T)$ are also called weights. $R$ is the root system corresponding to the pair $(G, T)$ and $R^{+}$is the 
set of positive roots. Choose a Borel subgroup $B$ such that all roots in $B$ are negative roots. There is a partial order in $X(T)$ such that $\lambda \geq \mu$ if $\lambda-\mu$ is in $\mathbf{N} R^{+}$. The Weyl group $W=N(T) / T$ acts on $X(T)$ in a natural way. There is a $W$-invariant inner product $\langle$,$\rangle on X(T) \otimes \mathbf{R}$ and $W$ is generated by the reflections $s_{\alpha}\left(\alpha \in R^{+}\right)$defined via $s_{\alpha}(\lambda)=\lambda-\left\langle\lambda, \alpha^{\vee}\right\rangle \alpha \quad\left(\alpha^{\vee}\right.$ is the coroot of $\alpha)$. The "dot" action of $W$ on $X(T)$ is defined by $w \cdot \lambda=w(\lambda+\rho)-\rho$ $(\forall \lambda \in X(T))$. Here $\rho=\frac{1}{2} \sum_{\alpha \in R^{+}} \alpha$.

The affine Weyl group $W_{p}$ acting on $X(T)$ is generated by all $s_{\alpha, n} \quad\left(\alpha \in R^{+}\right.$ and $n \in \mathbf{Z})$ such that $s_{\alpha, n} \cdot \lambda=s_{\alpha} \cdot \lambda+n p \alpha$ for all $\lambda \in X(T)$. Let $\lambda$, $\mu \in X(T)$. We say $\lambda$ is linked to $\mu$ if $\lambda \in W_{p} \cdot \mu$. We say $\lambda$ is strongly linked to $\mu$, denoted by $\lambda \uparrow \mu$, if either $\lambda=\mu$ or there is a sequence of weights $\lambda=\lambda_{0}, \lambda_{1}, \ldots, \lambda_{k}=\mu$ such that $\lambda_{i-1}=s_{\alpha_{i}, n_{i}} \cdot \lambda_{i}$ with some positive roots $\alpha_{i}$ and $\left\langle\lambda_{i}+\rho, \alpha_{i}^{\vee}\right\rangle>n_{i} p$.

Let $G_{r} T$ be the pullback of $T$ in $G$ via the $r$ th Frobenius homomorphism and $B_{r} T$ the pullback of $T$ in $B$. For each $\mu \in X(T)$, we denote

$$
Z_{r}^{\prime}(\mu)=\operatorname{Ind}_{B_{r} T}^{G_{r} T} \mu \text { and } Z_{r}(\mu)=\operatorname{Coind}_{B_{r}^{+} T}^{G_{r} T} \mu .
$$

Let $L_{r}(\mu)$ denote the unique simple submodule of $Z_{r}^{\prime}(\mu)$, which is also the unique simple quotient of $Z_{r}(\mu)$. By $Q_{r}(\mu)$, we denote the injective hull, which is also the projective cover, of $L_{r}(\mu)$ in the category $\mathscr{U}$ of all finite dimensional $G_{r} T$-modules. By the strong linkage principle, any two simple factors $L_{r}(\lambda)$ and $L_{r}(\mu)$ occurring as composition factors in an indecomposable module satisfy $\lambda \in W_{p} \cdot \mu$. We denote by $\mathscr{U}_{\mu}$ the category of $G_{r} T$ modules whose composition factors have all of their highest weights in $W_{p} \cdot \mu$.

Recall that $Z_{r}(\mu)$ and $Z_{r}^{\prime}(\mu)$ are indecomposable and all of their composition factors have highest weights strongly linked to $\mu$. We will refer to Jantzen's book [13] for unexplained facts or notations.

\section{TRUNCATED CATEGORIES}

1.1. Let us fix a weight $\lambda$ in $X(T)$. The category $\mathscr{U}_{\lambda}$ is an Abelian category. Let $\mathscr{U}_{t}(\lambda)$ be the full subcategory of $\mathscr{U}_{\lambda}$ such that each object of $\mathscr{U}_{t}(\lambda)$ has only those simple factors whose highest weights are strongly linked to $\lambda$. We call $\mathscr{U}_{t}(\lambda)$ a truncated category in $\mathscr{U}_{\lambda}$. It is easily seen that $\mathscr{U}_{t}(\lambda)$ is an Abelian category. Suppose $0 \rightarrow M \rightarrow N \rightarrow E \rightarrow 0$ is an exact sequence in $\mathscr{U}_{\lambda}$. Then $N$ is an object in $\mathscr{U}_{t}(\lambda)$ if and only if both $M$ and $E$ are objects in $\mathscr{U}_{t}(\lambda)$. In other words, $\mathscr{U}_{t}(\lambda)$ is a dense subcategory of $\mathscr{U}_{\lambda}$. The idea of constructing the truncated categories appears first in Donkin's work [5] for reductive groups. See $[6,3,4]$ for further discussion.

1.2. Let us denote the inclusion functor by $\tau: \mathscr{U}_{t}(\lambda) \rightarrow \mathscr{U}_{\lambda}$. Then $\tau$ is full, faithful, and exact. Next we construct its right and left adjoint functors and prove that they are left and right exact respectively.

Lemma. Let $M$ be an object in $\mathscr{U}_{\lambda}$. There is a unique maximal submodule $I(M)$ in $M$ and a unique quotient $P(M)$ such that both $I(M)$ and $P(M)$ are objects in $\mathscr{U}_{t}(\lambda)$.

Proof. Let $M_{1}$ and $M_{2}$ be submodules of $M$, which are objects in $\mathscr{U}_{t}(\lambda)$. Then the fact that $M_{1}+M_{2}$ is an object in $\mathscr{U}_{t}(\lambda)$ follows from the following exact sequence:

$$
0 \rightarrow M_{1} \rightarrow M_{1}+M_{2} \rightarrow M_{2} /\left(M_{1} \cap M_{2}\right) \rightarrow 0
$$


Therefore $I(M)$ is uniquely determined. To prove the uniqueness of $P(M)$, let us consider two submodules $N_{1}$ and $N_{2}$ such that both $M / N_{1}$ and $M / N_{2}$ are objects in $\mathscr{U}_{t}(\lambda)$. We show that $M /\left(N_{1} \cap N_{2}\right)$ is also an object in $\mathscr{U}_{t}(\lambda)$. It follows from the exact sequence

$$
0 \rightarrow N_{2} /\left(N_{1} \cap N_{2}\right) \rightarrow M /\left(N_{1} \cap N_{2}\right) \rightarrow M / N_{2} \rightarrow 0
$$

that it suffices to show $N_{2} /\left(N_{1} \cap N_{2}\right)$ is an object in $\mathscr{U}_{t}(\lambda)$. However, $N_{2} /\left(N_{1} \cap N_{2}\right) \subseteq M / N_{1}$. We are done.

Remarks. (1) By the definitions of $I(M)$ and $P(M)$ for each $M$ in $\mathscr{U}_{\lambda}$, we can see the following universal properties of $I(M)$ and $P(M)$ : For any object $N$ in $\mathscr{U}_{t}(\lambda)$ and any map $f: N \rightarrow M$ (or $f: M \rightarrow N$ ) in $\mathscr{U}_{\lambda}$, there is a unique map $g: N \rightarrow I(M)$ (or $g: P(M) \rightarrow N$ ) such that $f$ factors through $G$.

(2) $I$ and $P$ (if there is any confusion, we will add the subscript $\lambda$ to identify that they are related to the category $\left.\mathscr{U}_{t}(\lambda)\right)$ are well defined covariant functors from $\mathscr{U}_{\lambda}$ to $\mathscr{U}_{t}(\lambda)$.

Proposition. $I$ is the right adjoint functor and $P$ is the left adjoint functor of $\tau$. Proof. This is easily seen from the remarks above.

1.3. Let us recall that the duality functor $D: \mathscr{U}_{\lambda} \rightarrow \mathscr{U}_{\lambda}$ is defined as follows: Let $\sigma: G \rightarrow G$ be the automorphism corresponding to the automorphism of the root system: $\alpha \mapsto-\alpha$. It is clear that $\sigma$ induces an automorphism of the subgroup scheme $G_{r} T$. Then for each $G_{r} T$-module $M, D(M)$ is defined to be the module $\left(M^{*}\right)^{\sigma}$. Here $M^{*}$ is the dual module of $M$. The functor $D$ is exact, contravariant, and has the following properties:

(i) $D^{2}=$ id

(ii) $\operatorname{ch} D(M)=\operatorname{ch} M$ for all module $M$ in $\mathscr{U}_{\lambda}$,

(iii) $D L_{r}(\mu)=L_{r}(\mu)$,

(iv) $D \operatorname{Soc}^{i} M=\operatorname{Cap}^{i} D(M)$.

Here $\operatorname{Soc}^{i} M$ and $\operatorname{Rad}^{i} M$ are defined as usual and $\operatorname{Cap}^{i} M=M / \operatorname{Rad}^{i} M$, while $\operatorname{ch} M$ is the character of $M$ regarded as a $T$-module. If we set $\operatorname{Soc}_{i} M$ $=\operatorname{Soc}^{i} M / \operatorname{Soc}^{i-1} M$ and $\operatorname{Cap}^{i} M=\operatorname{Rad}^{i-1} M / \operatorname{Rad}^{i} M$, then $D \operatorname{Soc}_{i} M=$ $\mathrm{Cap}_{i} D M$.

It is easily seen from (ii) and (iii) that a module $M$ in $\mathscr{U}_{\lambda}$ is in $\mathscr{U}_{t}(\lambda)$ if and only if $D(M)$ is. So the restriction of $D$ to $\mathscr{U}_{t}(\lambda)$ is also exact and sends objects in $\mathscr{U}_{t}(\lambda)$ to objects in $\mathscr{U}_{t}(\lambda)$.

Proposition. $D \circ P=I \circ D$ and $P \circ D=D \circ I$.

Proof. We only prove the first equality. Let us consider the exact sequence $M \rightarrow P(M) \rightarrow 0$. Applying the exact functor $D$ to it one gets $0 \rightarrow D(P(M)) \rightarrow$ $D(M)$. As $D(P(M))$ is an object in $\mathscr{U}_{t}(\lambda)$, there is a unique injective map $f: D(P(M)) \rightarrow I(D(M))$. Applying the functor $D$ once again we get the following commutative diagram:

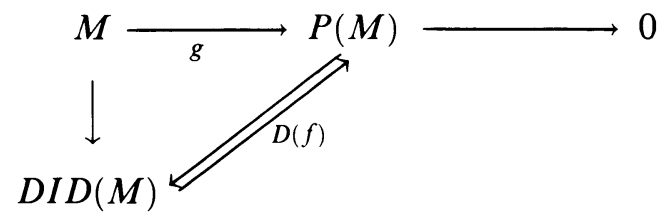


Here $D(f)$ is onto since $f$ is injective. However, the maximality of $P(M)$ implies that there is a unique map $g: P(M) \rightarrow D I D(M)$ such that the above diagram commutes. Note that $g$ has to be onto as the vertical map is onto. Now one can show from the diagram that $g$ and $D(f)$ are inverse to one another.

1.4. It is natural to ask whether the functors $I$ and $P$ are exact or not. However, by the exactness of $D$ and Proposition 1.3, one can see that the exactness of $P$ and that of $I$ are equivalent. Unfortunately, $I$ is not right exact unless $\lambda$ is a special point as one can see by applying $I$ to the exact sequence

$$
Z_{r}^{\prime}\left(\lambda^{\#}\right) \rightarrow L_{r}(\lambda) \rightarrow 0 .
$$

Here, $\lambda^{\#}$ is the weight such that $Z_{r}^{\prime}\left(\lambda^{\#}\right)$ has simple cap $L_{r}(\lambda)$, and $I\left(Z_{r}^{\prime}\left(\lambda^{\#}\right)\right)=$ 0 .

Proposition. $I$ is left exact and $P$ is right exact.

Proof. The proposition follows from Proposition 1.2 and the following lemma, which is suggested by Jantzen.

Lemma. Let $T: \mathscr{A} \rightarrow \mathscr{B}$ and $S: \mathscr{B} \rightarrow \mathscr{A}$ be covariant functors between exact additive categories. If $T$ is left adjoint of $S$, then $T$ is right exact and $S$ is left exact.

Proof. We only prove the statement for $T$. Let $A_{1} \rightarrow A_{2} \rightarrow A_{3} \rightarrow 0$ be exact in $\mathscr{A}$. By 13.2.5 of [19], the exactness of $T\left(A_{1}\right) \rightarrow T\left(A_{2}\right) \rightarrow T\left(A_{3}\right) \rightarrow 0$ is equivalent to exactness of $0 \rightarrow \operatorname{Hom}_{\mathscr{B}}\left(T\left(A_{3}\right), B\right) \rightarrow \operatorname{Hom}_{\mathscr{B}}\left(T\left(A_{2}\right), B\right) \rightarrow$ $\operatorname{Hom}_{\mathscr{B}}\left(T\left(A_{1}\right), B\right)$ for all $B$. But the latter is equivalent to the exactness of $0 \rightarrow \operatorname{Hom}_{\mathscr{A}}\left(A_{3}, S(B)\right) \rightarrow \operatorname{Hom}_{\mathscr{A}}\left(A_{2}, S(B)\right) \rightarrow \operatorname{Hom}_{\mathscr{A}}\left(A_{1}, S(B)\right)$ by the adjointness of $(T, S)$. Now the last exactness follows from the left exactness of $\operatorname{Hom}_{\mathscr{A}}(?, S(B))$.

1.5. Recall that $\mathscr{U}_{\lambda}$ has enough injective objects and projective objects. It follows from Proposition 1.2 that $P$ sends projective objects to projective objects and $I$ sends injective objects to injective objects since the inclusion functor $\tau$ is exact.

Proposition. $\mathscr{U}_{t}(\lambda)$ has enough injective and projective objects.

Proof. Let $M$ be an object in $\mathscr{U}_{t}(\lambda)$ and $Q \rightarrow M \rightarrow 0$ be exact with $Q$ projective in $\mathscr{U}_{\lambda}$. Now the right exactness of $P$ gives the exact sequence $P(Q) \rightarrow M \rightarrow 0$ and $P(Q)$ is projective in $\mathscr{U}_{t}(\lambda)$ (but may not be projective in $\left.\mathscr{U}_{\lambda}\right)$ by the above argument. The other half can be done similarly.

Let $L_{r}(\nu)$ be a simple module in $\mathscr{U}_{t}(\lambda)$ and $Q_{r}(\nu)$ be its projective cover (and its injective hull as well) in $\mathscr{U}_{\lambda}$. Therefore $D Q_{r}(\nu)=Q_{r}(\nu)$. It follows from the above argument that $I Q_{r}(\nu)$ and $P Q_{r}(\nu)$ are injective and projective respectively in $\mathscr{U}_{t}(\lambda)$. We claim that $I Q_{r}(\nu)$ and $P Q_{r}(\nu)$ are the injective hull and the projective cover of $L_{r}(\nu)$ in $\mathscr{U}_{t}(\lambda)$ respectively if $L_{r}(\nu)$ is an object in $\mathscr{U}_{t}(\lambda)$. In fact, we have $\operatorname{Soc} I Q_{r}(\nu)=L_{r}(\nu)$ since $I Q_{r}(\nu) \subseteq Q_{r}(\nu)$. Cap $P Q_{r}(\nu)=L_{r}(\nu)$ can be seen in the similar way. So we denote by $I(\nu)$ and $P(\nu)$ the injective hull and the projective cover of $L_{r}(\nu)$ in $\mathscr{U}_{t}(\lambda)$ respectively.

Note that the functor $D: \mathscr{U}_{t}(\lambda) \rightarrow \mathscr{U}_{t}(\lambda)$ is exact, full, and faithful. Then we have $\operatorname{Hom}_{\mathscr{U}_{t}(\lambda)}(D N, D M)=\operatorname{Hom}_{\mathscr{U}_{t}(\lambda)}(M, N)$. Hence $N$ is injective if 
and only if $D N$ is projective. Now $D L_{r}(\nu)=L_{r}(\nu)$ implies $D I(\nu)=P(\nu)$. Therefore the theory for injectives can be interpreted for projectives via $D$.

\section{THE STRUCTURE OF INJECTIVES}

2.1. Jantzen [13, II 11.4] proved that, in the category $\mathscr{U}_{\lambda}$, the injective hull $Q_{r}(\nu)$ of $L_{r}(\nu)$ has a filtration

$$
0=M^{0} \subseteq M^{1} \subseteq \cdots \subseteq M^{k}=Q_{r}(\nu)
$$

such that $M^{i} / M^{i-1}=Z_{r}^{\prime}\left(\nu_{i}\right)$ and the number of times the factor $Z_{r}^{\prime}\left(\nu_{i}\right)$ appears in the filtration is exactly the number of times $L_{r}(\nu)$ appears in a composition series of $Z_{r}^{\prime}\left(\nu_{i}\right)$, i.e.,

$$
\left(Q_{r}(\nu): Z_{r}^{\prime}\left(\nu_{i}\right)\right)=\left[Z_{r}^{\prime}\left(\nu_{i}\right): L_{r}(\nu)\right]
$$

From now on, we say a module $M$ has a good filtration if it has a filtration with factors of the form $Z_{r}^{\prime}(\nu)$ and we use $\left(M: Z_{r}^{\prime}(\nu)\right.$ ) (which does not depend on the choice of the good filtration by [5]) to stand for the number of $Z_{r}^{\prime}(\nu)$ appearing in the filtration, and $[E: L]$ stands for the multiplicity of a simple module $L$ in a module $E$.

One may ask if this kind of reciprocity still holds in the category $\mathscr{U}_{t}(\lambda)$. If a weight $\nu$ is low enough relative to $\lambda$, then $I(\nu)=Q_{r}(\nu)$ and we do have the above reciprocity.

Lemma. If $\operatorname{Ext}_{G_{r} T}^{i}\left(Z_{r}^{\prime}\left(\nu_{1}\right), Z_{r}^{\prime}\left(\nu_{2}\right)\right) \neq 0$ for some $i \geq 0$, then $\nu_{2} \uparrow \nu_{1}$.

Proof. It follows from [18] that $\operatorname{Ext}_{G_{r} T}^{i}\left(L_{r}(\mu), Z_{r}^{\prime}\left(\nu_{2}\right)\right) \neq 0$ implies $\nu_{2} \uparrow \mu$. One can see that $\operatorname{Ext}_{G_{r} T}^{i}\left(Z_{r}^{\prime}\left(\nu_{1}\right), Z_{r}^{\prime}\left(\nu_{2}\right)\right) \neq 0$ implies $\operatorname{Ext}_{G_{r} T}^{i}\left(L_{r}(\mu), Z_{r}^{\prime}\left(\nu_{2}\right)\right) \neq 0$ for some $\mu$ with $\left[Z_{r}^{\prime}\left(\nu_{1}\right): L_{r}(\mu)\right] \neq 0$. Now the lemma follows from the strong linkage principle. One can also see [14] for the proof.

2.2. By using Lemma 2.1, we can arrange the filtration of $Q_{r}(\nu)$ in such a way that if $M^{i} / M^{i-1}=Z_{r}^{\prime}\left(\nu_{i}\right)$, then $\nu_{i} \uparrow \nu_{j}$ implies $i \leq j$.

Proposition. $I(\nu)$ has a good filtration in $\mathscr{U}_{t}(\lambda)$ and

$$
\left(I(\nu): Z_{r}^{\prime}(\mu)\right)=\left[Z_{r}^{\prime}(\mu): L_{r}(\nu)\right] .
$$

Proof. Let $K$ be the submodule of $Q_{r}(\nu)$ with a good filtration which is a part of a good filtration of $Q_{r}(\nu)$ such that $K$ contains all and only those factors $Z_{r}^{\prime}\left(\nu_{i}\right)$ of $Q_{r}(\nu)$ with $\nu_{i} \uparrow \lambda$. The existence of $K$ can be easily seen from Lemma 2.1. Therefore, $Q_{r}(\nu) / K$ does not contain any factor $Z_{r}^{\prime}\left(\nu_{i}\right)$ with $\nu_{i} \uparrow \lambda$. It follows from the definition of $K$ and the strong linkage principle that $K$ is an object in the category $\mathscr{U}_{t}(\lambda)$. Thus $K \subseteq I(\nu)$ by the maximality of $I(\nu)$ in $Q_{r}(\nu)$. We claim that the equality holds.

Suppose $0=M^{0} \subseteq M^{1} \subseteq \cdots \subseteq M^{k}=Q_{r}(\nu)$ is a filtration such that $M^{i}=K$ for some $i . I(\nu) \neq K$ implies that there is a $j>i$ such that $I(\nu) \subseteq M^{j}$ and $I(\nu) \nsubseteq M^{j-1}$. Let $M^{j} / M^{j-1}=Z_{r}^{\prime}\left(\nu_{j}\right)$. Then $\nu_{j}$ is not strongly linked to $\lambda$ by the definition of $K$. But we have a nonzero homomorphism $I(\nu) \rightarrow Z_{r}^{\prime}\left(\nu_{j}\right)$. So $L_{r}\left(\nu_{j}\right)$ has to be a composition factor of $I(\nu)$ since it is the unique simple submodule of $Z_{r}^{\prime}\left(\nu_{j}\right)$. This is a contradiction because $I(\nu)$ is an object in $\mathscr{U}_{t}(\lambda)$. Hence $I(\nu)=K$ and $I(\nu)$ has a good filtration.

To show the reciprocity, we recall that $Z_{r}^{\prime}(\mu)$ is an object in $\mathscr{U}_{t}(\lambda)$ if and only if $\mu \uparrow \lambda$. With the above construction we have $\left(Q_{r}(\nu): Z_{r}^{\prime}(\mu)\right)=$ 
$\left(K: Z_{r}^{\prime}(\mu)\right)$ for all weights $\mu$ strongly linked to $\lambda$. So the equality in the theorem follows from the reciprocity for $Q_{r}(\nu)$ in $\mathscr{U}_{\lambda}$.

Remarks. (1) Similar results for reductive groups has been obtained by Donkin [5, (3.2), (12.1.6), (12.1.9)]. For more general categorical results, one can see [4]. One can also follow Donkin's argument to get the proof for the above proposition also. The proof given here follows from Irving's argument in [11], which gives another way to characterize the module $I(\nu)$.

(2) In order to consider the filtration for the projective covers of simple modules, we recall that $Z_{r}(\nu)=\operatorname{Coind}_{B_{r}^{+} T}^{G_{r} T} \nu \cong Z_{r}^{\prime}\left(w_{0}, \nu-2\left(p^{r}-1\right) \rho\right)$ and $D Z_{r}^{\prime}(\nu)=Z_{r}(\nu)$. So a similar proposition can be stated for the projective modules.

Corollary. $I(\lambda)=Z_{r}^{\prime}(\lambda)$.

Proof. Let $\mu \uparrow \lambda$ with $\left(I(\lambda): Z_{r}^{\prime}(\mu)\right) \neq 0$. Then $\left[Z_{r}^{\prime}(\mu): L_{r}(\lambda)\right] \neq 0$ and $\lambda \uparrow \mu$. This forces $\mu=\lambda$. Now the corollary follows from the fact $\left[Z_{r}^{\prime}(\lambda): L_{r}(\lambda)\right]=1$ and the proposition.

Remark. By using this corollary and Lemma 2.1 we can see that the exact sequence $0 \rightarrow Z_{r}^{\prime}(\mu) \rightarrow M \rightarrow Z_{r}^{\prime}(\lambda)-0$ splits if $L_{r}(\lambda)$ is a submodule of $M$.

2.3. In the rest of this section, we study the socle structure of the injective modules $I(\nu)$. Thanks to Proposition 2.2, $I(\nu)$ has a good filtration, so the method used for $Q_{1}(\nu)$ by Andersen and Kaneda in [2] can be used here. Let us recall some facts from [2].

For a module $M$, we denote by $[M]=\sum[M: L] L$ the element corresponding to $M$ in the Grothendieck group as well as the character of $M$, by understanding $\operatorname{ch}(M)=\sum[M: L] \operatorname{ch}(L)$. Here the sum is taken over all simple modules. We shall write $[M] \geq[N]$ if $[M: L] \geq[N: L]$ for all simple modules $L$.

Suppose $0 \rightarrow N \stackrel{j}{\rightarrow} M \stackrel{\pi}{\rightarrow} E \rightarrow 0$ is exact. If $\pi\left(\operatorname{Soc}^{j_{0}} M\right)=0$, then

$$
\left[\operatorname{Soc}^{j} N\right]+\left[\operatorname{Soc}^{j-j_{0}} E\right] \geq\left[\operatorname{Soc}^{j} M\right] .
$$

By applying the functor $D$, we have

$$
\left[\mathrm{Cap}^{j} E\right]+\left[\mathrm{Cap}^{j-j_{0}} N\right] \geq\left[\mathrm{Cap}^{j} M\right]
$$

if $\mathrm{Cap}^{j_{0}} M=\mathrm{Cap}^{j_{0}} E$ (i..e, $N \subseteq \operatorname{Rad}^{j_{0}} M$ ).

Let us fix a good filtration $0=M^{0} \subseteq M^{1} \subseteq \cdots \subseteq M^{k}=I(\nu)$ with $M^{i} / M^{i-1}=Z_{r}^{\prime}\left(\nu_{i}\right)$ such that $\nu_{i} \uparrow \nu_{j}$ implies $i \leq j$. Let us also fix a weight $\mu$ such that $\mu \uparrow \lambda$. Set $s=\max \left\{i \mid \nu_{i}=\mu\right\}$. Then $\left(I(\nu): Z_{r}^{\prime}(\mu)\right)=\left(M^{s}: Z_{r}^{\prime}(\mu)\right)$. The following lemma is analogous to Lemma 3.5 in [2].

Lemma. (i) $\left[M^{s}: L_{r}(\mu)\right]=\left[Z_{r}^{\prime}(\mu): L_{r}(\nu)\right]$.

(ii) $\left[\operatorname{Soc}^{j} M^{s}: L_{r}(\mu)\right]=\left[\operatorname{Soc}^{j} Z_{r}^{\prime}(\mu): L_{r}(\nu)\right]$.

Proof. The proof is similar to that of Lemma 3.5 in [2]. However, the injective module $I(\nu)$ may fail to be projective. So we give a proof of (i) here.

Note that $P(\mu)$ is the projective cover of $L_{r}(\mu)$ in $\mathscr{U}_{t}(\lambda)$. We have

$$
\begin{aligned}
{\left[M^{s}: L_{r}(\mu)\right] } & =\operatorname{dim} \operatorname{Hom}_{G_{r} T}\left(P(\mu), M^{s}\right) \\
& =\operatorname{dim}\left\{f \in \operatorname{Hom}_{G_{r} T}(P(\mu), I(\nu)) \mid \text { no weight of } \operatorname{Im}(f) \text { is }>\mu\right\}, \\
{\left[Z_{r}^{\prime}(\mu): L_{r}(\nu)\right] } & =\operatorname{dim} \operatorname{Hom}_{G_{r} T}\left(P(\nu), Z_{r}^{\prime}(\mu)\right) \\
& =\operatorname{dim}\left\{f \in \operatorname{Hom}_{G_{r} T}(P(\nu), I(\mu)) \mid \text { no weight of } \operatorname{Im}(f) \text { is }>\mu\right\} .
\end{aligned}
$$


Now $f \mapsto D(f)$ defines an isomorphism

$$
\operatorname{Hom}_{G_{r} T}(P(\mu), I(\nu)) \rightarrow \operatorname{Hom}_{G_{r} T}(P(\nu), I(\mu)) .
$$

Keeping in mind that $\operatorname{Im}(D(f))=D(\operatorname{Im}(f))$, we have

$$
\operatorname{ch}(\operatorname{Im}(f))=\operatorname{ch}(\operatorname{Im}(D(f))) .
$$

Therefore, this isomorphism induces an isomorphism between the above described subspaces and the equality in (i) follows.

2.4. Proposition. For any $m>0$ and $\nu \uparrow \lambda$, we have

$$
\left[\operatorname{Soc}^{m} I(\nu)\right] \leq \sum_{\mu \uparrow \lambda} \sum_{j}\left[\operatorname{Soc}_{j} Z_{r}^{\prime}(\mu): L_{r}(\nu)\right]\left[\operatorname{Soc}^{m+1-j} Z_{r}^{\prime}(\mu)\right] \text {. }
$$

Proof. As we mentioned before, $Z_{r}^{\prime}(\mu)$ is an object in $\mathscr{U}_{t}(\lambda)$ if and only if $\mu \uparrow \lambda$. We can copy the proof given by Andersen and Kaneda in [2, 3.6-3.7].

2.5. Let $\nu, \mu \in W_{p} \cdot \lambda$ and $\nu \uparrow \lambda, \mu \uparrow \lambda$. The following result is an analogue of a result of Irving for category $\mathscr{O}^{\lambda}$ [10]. The first version of this result was due to Landrock for the group algebras of finite groups.

Theorem. For any $i \geq 1$, we have

$$
\left[\operatorname{Soc}_{i} I(\nu): L_{r}(\mu)\right]=\left[\operatorname{Soc}_{i} I(\mu): L_{r}(\nu)\right] .
$$

Proof. It is enough to show $\left[\operatorname{Soc}^{i} I(\nu): L_{r}(\mu)\right]=\left[\operatorname{Soc}^{i} I(\mu): L_{r}(\nu)\right]$. Note that $[M: L]=[D M: L]$ for any module $M$ and simple module $L$. Since $D\left(\operatorname{Soc}^{i} I(\mu)\right)=\operatorname{Cap}^{i} D I(\mu)=\operatorname{Cap}^{i} P(\mu)$,

$\left[\operatorname{Soc}^{i} I(\mu): L_{r}(\nu)\right]=\left[\operatorname{Cap}^{i} P(\mu): L_{r}(\nu)\right]=\operatorname{dim} \operatorname{Hom}_{G_{r} T}\left(\operatorname{Cap}^{i} P(\mu), I(\nu)\right)$.

Now the theorem follows from the fact that

$$
\operatorname{Hom}_{G_{r} T}\left(\operatorname{Cap}^{i} M, N\right)=\operatorname{Hom}_{G_{r} T}\left(M, \operatorname{Soc}^{i} N\right)
$$

for any $G_{r} T$-modules $N$ and $M$.

2.6. One may ask whether the inequality given in Proposition 2.4 is an equality or not. The following result gives some information toward a positive answer.

Corollary. Let $\nu, \mu \uparrow \lambda$. If $\left[\operatorname{Soc}_{i} Z_{r}^{\prime}(\nu): L_{r}(\mu)\right]=t$, then there are submodules $U \subseteq V$ of $I(\mu)$ with good filtrations such that $V / U=Z_{r}^{\prime}(\nu)^{\oplus t}$ and

$$
\left[\operatorname{Soc}_{i} V: L_{r}(\nu)\right]=t \text { and }\left[\operatorname{Soc}_{i} U: L_{r}(\nu)\right]=0 \text {. }
$$

Proof. $\nu \uparrow \lambda$ implies that $\mathscr{U}_{t}(\nu)$ is a full subcategory of $\mathscr{U}_{t}(\lambda)$. We denote by $I_{\nu}^{\lambda}$ the restriction of the functor $I_{\nu}: \mathscr{U}_{\lambda} \rightarrow \mathscr{U}_{t}(\nu)$ to $\mathscr{U}_{t}(\lambda)$. Then we have $I_{\nu}=I_{\nu}^{\lambda} \circ I_{\lambda}$. Especially we get $Z_{r}^{\prime}(\nu)=I_{\nu}\left(Q_{r}(\nu)\right)=I_{\nu}^{\lambda}(I(\nu))$ (we still use $I(\nu)$ to denote the injective object in $\left.\mathscr{U}_{t}(\lambda)\right)$ and $I_{\nu}^{\lambda} I(\mu)$ is the injective hull of $L_{r}(\mu)$ in the category $\mathscr{U}_{t}(\nu)$. Now we can apply the formula of Theorem 2.5 to the category $\mathscr{U}_{t}(\nu)$ and get

$$
\left[\operatorname{Soc}_{i} I_{\nu}^{\lambda} I(\mu): L_{r}(\nu)\right]=\left[\operatorname{Soc}_{i} Z_{r}^{\prime}(\nu): L_{r}(\mu)\right] .
$$

Recall that $I_{\nu}^{\lambda} I(\mu)$ has a good filtration and all of its factors have the form $Z_{r}^{\prime}(\eta)$ with $\eta \uparrow \nu$. Thus each copy of $L_{r}(\nu)$ has to occur in a factor $Z_{r}^{\prime}(\nu)$ of $I_{\nu}^{\lambda} I(\mu)$. We can choose a good filtration $0=M^{0} \subseteq M^{1} \subseteq \cdots \subseteq M^{s}=I_{\nu}^{\lambda} I(\mu)$ such that the smallest number $j$ with $\operatorname{Soc}^{i} I_{\nu}^{\lambda} I(\mu) \subseteq M^{j}$ in this filtration is the 
smallest one among all good filtrations of $I_{\nu}^{\lambda} I(\mu)$. Now let $V=M^{j}$. Let $U_{0}$ be defined such that $\operatorname{Soc}^{i} I_{\nu}^{\lambda} I(\mu) / U_{0}=L_{r}(\nu)^{\oplus t}$. We can define $U$ from $U_{0}$ in $V$ in the same way as we define $V$ from $\operatorname{Soc}^{i} I_{\nu}^{\lambda} I(\mu)$ in $I_{\nu}^{\lambda} I(\mu)$. Then one can easily check the conditions for $U$ and $V$ by using the remark following the corollary in 2.2.

Remark. $I(\mu)$ is not rigid if $L_{r}(\mu)$ occurs in more than one socle layer in $Z_{r}^{\prime}(\lambda)$ according to the above proof, since one can put all factors of the form $Z_{r}^{\prime}(\lambda)$ on the top and therefore all the composition factors of the form $L_{r}(\lambda)$ will appear in only one radical layer.

2.7. Let $M$ be a module of finite length. We denote the Loewy length of $M$ by $l l(M)$, which is the smallest $i$ with $\operatorname{Soc}^{i} M=M$ and also the smallest $i$ with $\operatorname{Rad}^{i} M=0$. Here we can get some lower bound to the Loewy length of some special modules in $\mathscr{U}_{\lambda}$.

Corollary (A). If $\left[\operatorname{Soc}_{i} Z_{r}^{\prime}(\lambda): L_{r}(\mu)\right] \neq 0$, then $l l(I(\mu)) \geq i+l l\left(Z_{r}^{\prime}(\lambda)\right)-1$. Proof. Take $\lambda=\nu$ in Corollary 2.6.

For each weight $\mu$ in $X(T)$, we have a unique decomposition $\mu=\mu_{0}+p^{r} \mu_{1}$ with $\mu_{0} \in X_{r}(T)$ (the set of $p^{r}$-restricted weights).

Corollary (B). $l l\left(Q_{r}(\mu)\right) \geq 2 l l\left(Z_{r}^{\prime}\left(w_{0}\left(\mu_{0}\right)+p^{r} \mu_{1}+2\left(p^{r}-1\right) \rho\right)\right)-1$.

Proof. Let $\lambda=w_{0}\left(\mu_{0}\right)+p^{r} \mu_{1}+2\left(p^{r}-1\right) \rho$. If we can show $Q_{r}(\mu)=I(\mu)$ in the category $\mathscr{U}_{t}(\lambda)$, we are done by Corollary (A). So we only need to show that all factors $Z_{r}^{\prime}(\eta)$ of $Q_{r}(\mu)$ satisfy $\eta \uparrow \lambda$. Since $Q_{r}(\mu)$ has the unique simple quotient $L_{r}(\mu), Z_{r}^{\prime}(\lambda)$ has to appear in the top for any good filtration of $Q_{r}(\mu)$. If there is an $\eta$ with $\left[Z_{r}^{\prime}(\mu): L_{r}(\eta)\right] \neq 0$ such that $\eta$ is not strongly linked to $\lambda$, we can use the extension property in 2.1 to show that $Q_{r}(\mu)$ has a good filtration with $Z_{r}^{\prime}\left(\eta^{\prime}\right)$ on the top for some weight $\eta^{\prime}$ such that $\eta^{\prime}$ is not strongly linked to $\lambda$. This is a contradiction and the corollary follows.

Remark. For $r=1$, this result has been proved by Andersen and Kaneda [2].

\section{TRANSLATION FUNCTORS}

As we have seen in Corollary 2.2, $I(\lambda)$ can be characterized by some induced modules. In this section we can see that the injective hulls of the simple modules with highest weights close to $\lambda$ and "around" a $p$-singular weight behave like the projective modules in the category $\mathscr{O}$ for a complex semisimple Lie algebra and the induced modules are like the Verma module with a possibly "smaller" root system.

3.1. Recall that the affine Weyl group $W_{p}$ acting on $X(T) \otimes \mathbf{R}$ is generated by the reflections $s_{\alpha, n p}$ in the hyperplanes $H_{\alpha, n p}=\left\{x \in X(T) \otimes \mathbf{R} \mid\left\langle x+\rho, \alpha^{\vee}\right\rangle=n p\right\}$ $(\alpha \in R$ and $n \in \mathbf{Z})$. The connected components of the complement of $\bigcup H_{\alpha, n p}$ in $X(T) \otimes \mathbf{R}$ are called alcoves. The closure of an alcove is a fundamental domain of the $W_{p}$-action on $X(T) \otimes \mathbf{R}$. Each facet as defined in [13, II 6.2] is contained in the closure of an alcove. With the chosen positive root system, we can talk about the upper closure of a facet as in [13, II 6.2]. We will use $\widehat{F}$ or $\widehat{A}$ to denote the upper closure of a facet $F$ or an alcove $A$.

Let $\mu \in X(T)$ and write $\mu=\mu_{0}+p^{r} \mu_{1}$ with $\mu_{0} \in X_{r}(T)$. We can define a new action of $W_{p}$ on $X(T)$ by $w_{r} \cdot \mu=\mu_{0}+p^{r} w \cdot \mu_{1}$ for all $w \in W_{p}$ and 
$\mu \in X(T)$. For each $\mu \in X(T)$, we use $W_{\mu}$ to denote the stabilizer of $\mu$ in $W_{p}$. Then $W_{\mu}$ is a finite subgroup of $W_{p}$ and is generated by reflections with the fixed hyperplanes going through $\mu$. One can view $W_{p}$ as a Coxeter group acting on the set of alcoves, so that $W_{\mu}$ (as the stabilizer of the set of alcoves containing $\mu$ in their closures) is a parabolic subgroup of $W_{p}$. Then the restriction of the length function $l(\cdot)$ of $W_{p}$ to $W_{\mu}$ is the length function of $W_{\mu}$ on its own. We will use $w_{\mu}$ to denote the unique longest element of $W_{\mu}$. If $A$ is an alcove containing $\mu$ in its closure, there is a unique alcove $A_{\mu}^{-}$which contains $\mu$ in its upper closure and is minimal in the set $W_{\mu} \cdot A$ (the order defined on the set of all alcoves is consistent with the strong linkage order). So $A_{\mu}^{+}=w_{\mu} \cdot A_{\mu}^{-}$is the unique maximal one. We call a weight $\mu$ a $p^{r}$-special point if $\mu=p^{r} \nu-\rho$ for some $\nu$ in $X(T)$. In the rest of this paper we assume $p \geq h$ ( $h$ is the Coxeter number of the root system), so that each alcove contains an integral weight.

3.2. Our motivation is the following. If $\mu$ is a $p^{r}$-special point and $\lambda$ is a weight in $A_{\mu}^{+}$, then the module $T_{\mu}^{\lambda} Z_{r}^{\prime}(\mu)$ is the injective hull of $L_{r}\left(w_{\mu} \cdot \lambda\right)$ in the category $\mathscr{U}_{\lambda}$. (We abuse the notation here: we will simply use the weights $\lambda$ and $\mu$ to denote the translation functor instead of their images in the bottom alcove under the $W_{p}$-action.) This is true because $Z_{r}^{\prime}(\mu)$ is injective in the category $\mathscr{U}_{\mu}$. This is not true if $\mu$ is not a $p^{r}$-special point. However this is the case if we consider them in a suitably smaller category.

Let $\mu$ be a weight with the stabilizer $W_{\mu}$ in $W_{p}$. Let $\lambda$ be a weight in a facet $F$ such that $\bar{F}$ (the closure of $F$ ) contains $\mu$. Clearly we have $W_{\mu} \supseteq W_{\lambda}$. The set $W_{\mu} \cdot \lambda$ possesses a unique minimal weight denoted by $\lambda_{\#}$ and a unique maximal weight with respect to the strong linkage order. We will assume that $\lambda$ is the maximal one.

With the above notation we need to ask if the translation functors $T_{\mu}^{\lambda}$ and $T_{\lambda}^{\mu}$ are defined between $\mathscr{U}_{t}(\mu)$ and $\mathscr{U}_{t}(\lambda)$.

Lemma. (i) If $M$ is an object in $\mathscr{U}_{t}(\mu)$, then $T_{\mu}^{\lambda} M$ is an object in $\mathscr{U}_{t}(\lambda)$.

(ii) If $N$ is an object in $\mathscr{U}_{t}(\lambda)$, then $T_{\lambda}^{\mu} N$ is an object in $\mathscr{U}_{t}(\mu)$.

Proof. It suffices to prove the lemma for simple modules. (i) Suppose $y \cdot \mu \uparrow \mu$ with $y \in W_{p}$. We need to show that all composition factors of $T_{\mu}^{\lambda} L_{r}(y \cdot \mu)$ are in $\mathscr{U}_{t}(\lambda)$. It is enough to show that all the weights in the set $\left\{y w \cdot \lambda \mid w \in W_{\mu}\right\}$ are strongly linked to $\lambda$ since $T_{\mu}^{\lambda} L_{r}(y \cdot \mu) \subseteq T_{\mu}^{\lambda} Z_{r}^{\prime}(y \cdot \mu)$ and

$$
\operatorname{ch} T_{\mu}^{\lambda} Z_{r}^{\prime}(y \cdot \mu)=\sum_{w \in W_{\mu} / W_{\lambda}} \operatorname{ch} Z_{r}^{\prime}(y w \cdot \lambda) .
$$

If we can show that $y \cdot \lambda^{\prime} \uparrow \lambda^{\prime}$ for all $\lambda^{\prime} \in W_{\mu} \cdot \lambda$ we are done since $\lambda$ is maximal. Further we can assume that $y=s_{\alpha, n p}$ for some $\alpha \in R^{+}$and $\left\langle\mu+\rho, \alpha^{\vee}\right\rangle>n p$. Now $\mu$ is in the closure of the facet containing $\lambda^{\prime}$. We must have $\left.\left\langle\lambda^{\prime}+\rho, \alpha^{\vee}\right\rangle\right\rangle$ $n p$. This proves (i).

(ii) This is easier than (i) since $T_{\lambda}^{\mu} L_{r}(y \cdot \lambda)=L_{r}(y \cdot \mu)$ if $y \cdot \mu$ is in the upper closure of the facet containing $y \cdot \lambda$ and 0 otherwise. We need to show $y \cdot \mu \uparrow \mu$ if $y \cdot \lambda \uparrow \lambda$. Similarly we may assume that $y=s_{\alpha, n p}$ with $\left\langle\lambda+\rho, \alpha^{\vee}\right\rangle>n p$. So we have $\left\langle\mu+\rho, \alpha^{\vee}\right\rangle \geq n p$ and we are done. 
Corollary. (i) $T_{\mu}^{\lambda}$ and $T_{\lambda}^{\mu}$ are a pair of adjoint functors between $\mathscr{U}_{t}(\lambda)$ and $\mathscr{U}_{t}(\mu)$.

(ii) $T_{\lambda}^{\mu}$ and $T_{\mu}^{\lambda}$ send injective (projective) modules to injective (projective) modules.

3.3. In this subsection we assume that $\lambda$ and $\mu$ as in 3.2. If $M$ is a module in $\mathscr{U}_{t}(\mu)$ with simple $\operatorname{Cap}^{1}(M)$, then the adjointness of the pair $\left(T_{\lambda}^{\mu}, T_{\mu}^{\lambda}\right)$ shows that $\operatorname{Cap}^{1}\left(T_{\mu}^{\lambda} M\right)$ is simple and, therefore, $T_{\mu}^{\lambda} M$ is indecomposable in $\mathscr{U}_{t}(\lambda)$. In general, $T_{\mu}^{\lambda} M$ is indecomposable if $M$ is indecomposable in $\mathscr{U}_{t}(\mu)$. In fact, $M$ is a sum of submodules $N_{i}(i=1,2, \ldots, t)$ with simple $\operatorname{Cap}^{1}\left(N_{i}\right)$ and nonzero image under the quotient map $M \rightarrow \operatorname{Cap}^{1}(M) . M$ is indecomposable implies that any two such submodules have a nonzero intersection. Since an exact functor preserves intersection and sum, all the submodules $T_{\mu}^{\lambda} N_{i}\left(T_{\mu}^{\lambda} M\right.$ is a sum of these submodules) are contained in the same indecomposable component of $T_{\mu}^{\lambda} M$ ( $T_{\mu}^{\lambda}$ is faithful), therefore $T_{\mu}^{\lambda} M$ is indecomposable. In particular, $T_{\mu}^{\lambda} Z_{r}^{\prime}(\mu)$ is indecomposable and has simple socle $L_{r}\left(\lambda_{\#}\right)$. On the other hand, $Z_{r}^{\prime}(\mu)$ is injective in $\mathscr{U}_{t}(\mu)$ by Corollary 2.2. Thus we have $T_{\mu}^{\lambda} Z_{r}^{\prime}(\mu)=I\left(\lambda_{\#}\right)$. Therefore all the arguments in $\S 2$ for injectives work for $T_{\mu}^{\lambda} Z_{r}^{\prime}(\mu)$. In particular, $T_{\mu}^{\lambda} Z_{r}^{\prime}(\mu)$ has a good filtration.

Proposition. If $Z_{r}^{\prime}(\nu)$ is in $\mathscr{U}_{t}(\lambda)$, i.e., $\nu \uparrow \lambda$. Then $\left[Z_{r}^{\prime}(\nu): L_{r}\left(\lambda_{\#}\right)\right]=1$ if $\nu \in W_{\mu} \cdot \lambda$ and 0 otherwise.

Proof. Let us recall that the translation functor $T_{\lambda}^{\mu}$ sends a simple module to a simple module or zero. Let $\nu=y \cdot \lambda_{\#}$ for some $y \in W_{p}$. Then $T_{\lambda}^{\mu} Z_{r}^{\prime}(\nu)=$ $Z_{r}^{\prime}(y \cdot \mu)$. The above discussion shows that $\left[T_{\lambda}^{\mu} Z_{r}^{\prime}(\nu): L_{r}(\mu)\right]=\left[Z_{r}^{\prime}(\nu): L_{r}\left(\lambda_{*}\right)\right]$. However $\left[Z_{r}^{\prime}(y \cdot \mu): L_{r}(\mu)\right] \neq 0$ implies $\mu \uparrow y \cdot \mu$. But by the proof of Lemma 3.2 we have $y \cdot \mu \uparrow \mu$. This forces $y \cdot \mu=\mu$ and $y \in W_{\mu}$. It is well known that $\left[Z_{r}^{\prime}(\mu): L_{r}(\mu)\right]=1$. Now the proposition follows.

Remark. The above result has been proved by Koppinen [15].

3.4. In this subsection we use the setup for the weights $\lambda$ and $\mu$ as in 3.2. But we assume that $\lambda$ is $p$-regular, i.e., $W_{\lambda}=\{1\}$ is trivial. In this case we can say something about the position of $L_{r}\left(\lambda_{\#}\right)$ in all $Z_{r}^{\prime}(w \cdot \lambda)$ with $w \in W_{\mu}$. Since $p \geq h$ as we assumed in 3.1, we can find an integral weight in each alcove wall by [13, II 6.3(1)]. Let $\lambda^{\prime}$ be a weight in $W_{\mu} \cdot \lambda$ and $F$ a wall of the alcove containing $\lambda^{\prime}$, such that $\mu \in \bar{F}$ and $\lambda^{\prime}>s \cdot \lambda^{\prime}$ if $s$ is the reflection in the hyperplane containing $F$. For each integral weight $\nu$ in $F$, we have a nonsplitting exact sequence

$$
0 \rightarrow Z_{r}^{\prime}\left(s \cdot \lambda^{\prime}\right) \rightarrow T_{\nu}^{\lambda^{\prime}} Z_{r}^{\prime}(\nu) \rightarrow Z_{r}^{\prime}\left(\lambda^{\prime}\right) \rightarrow 0
$$

and $\operatorname{dim} \operatorname{Hom}_{G_{r} T}\left(Z_{r}^{\prime}\left(\lambda^{\prime}\right), Z_{r}^{\prime}\left(s \cdot \lambda^{\prime}\right)\right)=1$. Consider the nonzero map $\phi: Z_{r}^{\prime}\left(\lambda^{\prime}\right)$ $\rightarrow Z_{r}^{\prime}\left(s \cdot \lambda^{\prime}\right)$, which kills the socle of $Z_{r}^{\prime}(\lambda)$. In this way, we can have a sequence of maps for each $w \in W_{\mu}$,

$$
Z_{r}^{\prime}\left(w \cdot \lambda_{\#}\right) \stackrel{\phi_{l(w)}}{\rightarrow} \cdots \stackrel{\phi_{1}}{\rightarrow} Z_{r}^{\prime}\left(\lambda_{\#)}\right)
$$

Let $\phi_{w}$ be the composite of the above maps. Then $\phi_{w}\left(\operatorname{Soc}^{l(w)} Z_{r}^{\prime}\left(w \cdot \lambda_{\#}\right)\right)=0$. By applying the translation functor $T_{\lambda}^{\mu}$ to this sequence of maps we see that the 
composition of these maps is not zero since each $T_{\lambda}^{\mu}\left(\phi_{i}\right)$ is an automorphism of $Z_{r}^{\prime}(\mu)\left(T_{\lambda}^{\nu}\left(\phi_{i}\right)\right.$ has already been seen to be an isomorphism for a suitable choice of $\nu$ ). Thus we have proved the following.

Lemma. (i) $\left[\operatorname{Soc}^{l(w)} Z_{r}^{\prime}\left(w \cdot \lambda_{\#}\right): L_{r}\left(\lambda_{\#}\right)\right]=0$;

(ii) $T_{\lambda}^{\mu} \operatorname{Soc}^{l(w)} Z_{r}^{\prime}\left(w \cdot \lambda_{\#}\right)=0$;

(iii) $l l\left(Z_{r}^{\prime}(\mu)\right) \leq l l\left(Z_{r}^{\prime}(\lambda)\right)-l\left(w_{\mu}\right)$.

3.5. Again we assume $\lambda$ to be $p$-regular.

Proposition. [ $\left.\operatorname{Soc}^{l(w)} T_{\mu}^{\lambda} Z_{r}^{\prime}(\mu): L_{r}\left(w \cdot \lambda_{\sharp}\right)\right]=0$ for all $w \in W_{\mu}$.

Proof. By Lemma 3.4, $\left[\operatorname{Soc}_{j} Z_{r}^{\prime}\left(w \cdot \lambda_{\#}\right): L_{r}\left(\lambda_{\#}\right)\right] \neq 0$ implies $j>l(w)$. Now using Proposition 3.3, the formula in 2.4 for $I\left(\lambda_{\#}\right)=T_{\mu}^{\lambda} Z_{r}^{\prime}(\mu)$ can be rewritten as

$$
\begin{aligned}
& {\left[\operatorname{Soc}^{n} I\left(\lambda_{\#}\right): L_{r}\left(w \cdot \lambda_{\#}\right)\right]} \\
& \quad \leq \sum_{y \in W_{\mu}} \sum_{j>l(y)}\left[\operatorname{Soc}_{j} Z_{r}^{\prime}\left(y \cdot \lambda_{\#}\right): L_{r}\left(\lambda_{\#}\right)\right]\left[\operatorname{Soc}^{n+1-j} Z_{r}^{\prime}\left(y \cdot \lambda_{\#}\right): L_{r}\left(w \cdot \lambda_{\#}\right)\right] .
\end{aligned}
$$

Now $\left[\operatorname{Soc}^{n+1-j} Z_{r}^{\prime}\left(y \cdot \lambda_{\#}\right): L_{r}\left(w \cdot \lambda_{\#}\right)\right] \neq 0$ implies that $l(y) \geq l(w)$ and $n-j \geq$ 0 . Therefore $n>l(w)$ and the proposition follows.

3.6. The following proposition resembles a result of Irving [9] for semisimple Lie algebras.

Proposition. Let $\lambda$ be p-regular and $\mu$ in the closure of the alcove containing $\lambda$. Then

(i) $l l\left(T_{\mu}^{\lambda} L_{r}(\mu)\right) \geq 1+2 l\left(w_{\mu}\right)$

(ii) For any module $M$ of finite length in $\mathscr{U}_{\mu}, l l\left(T_{\mu}^{\lambda} M\right) \geq l l(M)+2 l\left(w_{\mu}\right)$.

Proof. To prove (i) we assume that $\lambda$ is maximal in the set $W_{\mu} \cdot \lambda$ as in 3.2. Then we can consider the modules in the category $\mathscr{U}_{t}(\lambda)$. Now we have $T_{\mu}^{\lambda} Z_{r}^{\prime}(\mu)=I\left(\lambda_{\#}\right)$ and $T_{\mu}^{\lambda} L_{r}(\mu) \subseteq I\left(\lambda_{\#}\right)$. By Proposition 3.3 we get

$$
\left[T_{\mu}^{\lambda} L_{r}(\mu): L_{r}\left(\lambda_{\#}\right)\right]=\left[T_{\mu}^{\lambda} Z_{r}^{\prime}(\mu): L_{r}\left(\lambda_{\#}\right)\right]=\left|W_{\mu}\right| .
$$

However, $\operatorname{Soc}^{i} T_{\mu}^{\lambda} L_{r}(\mu)=T_{\mu}^{\lambda} L_{r}(\mu) \cap \operatorname{Soc}^{i} T_{\mu}^{\lambda} Z_{r}^{\prime}(\mu)$. If we denote $s=$ $l l\left(T_{\mu}^{\lambda} L_{r}(\mu)\right)$, we have the following:

$$
\begin{aligned}
\left|W_{\mu}\right| & =\left[\operatorname{Soc}^{s} T_{\mu}^{\lambda} Z_{r}^{\prime}(\mu): L_{r}\left(\lambda_{\#}\right)\right] \\
& \leq \sum_{w \in W_{\mu}} \sum_{j}\left[\operatorname{Soc}_{j} Z_{r}^{\prime}\left(w \cdot \lambda_{\#}\right): L_{r}\left(\lambda_{\#}\right)\right]\left[\operatorname{Soc}^{s+1-j} Z_{r}^{\prime}\left(w \cdot \lambda_{\#}\right): L_{r}\left(\lambda_{\#}\right)\right] .
\end{aligned}
$$

For each $w \in W_{\mu}$, there is only one $j_{w}>l(w)$ (as in 3.4) such that

$$
\left[\operatorname{Soc}_{j} Z_{r}^{\prime}\left(w \cdot \lambda_{\#}\right): L_{r}\left(\lambda_{\#}\right)\right]= \begin{cases}1 & \text { if } j=j_{w} \\ 0 & \text { otherwise. }\end{cases}
$$

If $\left[\operatorname{Soc}^{s+1-j} Z_{r}^{\prime}\left(w \cdot \lambda_{\#}\right): L_{r}\left(\lambda_{\#}\right)\right] \neq 0$, we have $s+1-j>l(w)$. This shows that

$$
\sum_{j}\left[\operatorname{Soc}_{j} Z_{j}^{\prime}\left(w \cdot \lambda_{\#}\right): L_{r}\left(\lambda_{\#}\right)\right]\left[\operatorname{Soc}^{s+1-j} Z_{r}^{\prime}\left(w \cdot \lambda_{\#}\right): L_{r}\left(\lambda_{\#}\right)\right]=1
$$


only if $s+1 \geq 2 l(w)+2$, i..e, $s \geq 1+2 l(w)$. Therefore

$$
\left|W_{\mu}\right|=\left[\operatorname{Soc}^{s} T_{\mu}^{\lambda} Z_{r}^{\prime}(\mu): L_{r}\left(\lambda_{\#}\right)\right]
$$

implies $s \geq 1+2 l\left(w_{\mu}\right)$ and (i) is proved.

To prove (ii), we may assume that $\operatorname{Cap} M$ is simple. Keepirg in mind that $T_{\mu}^{\lambda} K$ is indecomposable if $K$ is so, the argument is exactly the same as Irving's proof of Proposition 3.3(ii) in [9].

3.7. Let us introduce some notations. There is an involutive automorphism of the Abelian group $X(T)$ defined by $\lambda^{*}=-w_{0}(\lambda)$ (for all $\lambda \in X(T)$ ). We define the action $w_{r}(\lambda)=\lambda_{0}+p^{r} w\left(\lambda_{1}\right)$ according to the decomposition $\lambda=\lambda_{0}+p^{r} \lambda_{1}$. It is easy to check that $w_{r} \cdot \lambda \in W_{p} \cdot \lambda$ and $w_{r}(\lambda) \in W_{p} \cdot \lambda$. Further we set

$$
\bar{\lambda}=\lambda_{0}^{*}-p^{r} \lambda_{1}, \quad \tilde{\lambda}=2\left(p^{r}-1\right)-\lambda .
$$

Note that $\alpha \mapsto \alpha^{*}$ defines an automorphism of the root system and maps positive roots to positive roots. So it induces an automorphism of the group $W_{p}$ (and also of $W$ ). This automorphism of the group $W_{p}$ can be expressed via $y^{*} \cdot \lambda=\left(y \cdot\left(\lambda^{*}\right)\right)^{*}$ for $y \in W_{p}$. Thus we have the following properties:

(i) $(y \cdot \lambda)^{*}=y^{*} \cdot \lambda^{*}$ for $\lambda \in X(T)$ and $y \in W_{p}$,

(ii) $\left(s_{\alpha, n p}\right)^{*}=s_{\alpha^{*}, n p}$ for $\alpha \in R^{+}$and $n \in \mathbf{Z}$.

Note that $*$ sends simple roots to simple roots and highest short root to highest short root. So we get $l(y)=l\left(y^{*}\right)$ for all $y \in W_{p}$, i.e., the automorphism preserves the length function of the Coxeter group $W_{p}$. By using the definition, we can easily verify the following for $\lambda \in X(T)$ and $y \in W_{p}$.

$$
\bar{\lambda}=\left(w_{0}\right)_{r}\left(\lambda^{*}\right), \quad \overline{y \cdot \lambda}=y^{*} \cdot \bar{\lambda}, \quad \tilde{\lambda}=w_{0} \cdot \lambda^{*}+2 p^{r} \rho .
$$

If $F$ is a facet, $\lambda \in \widehat{F}$ if and only if $\lambda^{*} \in \widehat{F}^{*}$.

We can also define an automorphism of the group $W_{p}$ such that $\widetilde{y} \cdot \lambda=\tilde{y} \cdot \tilde{\lambda}$. Here $\tilde{y}=t_{2 p^{r} \rho} w_{0} y^{*} w_{0} t_{2 p^{r} \rho}^{-1}\left(t_{2 p^{r} \rho}\right.$ is the translation by $2 p^{r} \rho$ on $\left.X(T)\right)$.

Lemma. Let $\lambda, \mu \in X(T)$. Then

(i) $\lambda \in W_{p} \cdot \mu$ if and only if $\tilde{\lambda} \in W_{p} \cdot \tilde{\mu}$.

(ii) $*: W_{\lambda} \rightarrow W_{\lambda^{*}}$ and $\sim: W_{\lambda} \rightarrow W_{\tilde{\lambda}}$ are group isomorphisms and preserve the length functions.

(iii) $\tilde{\lambda}, \bar{\lambda} \in W_{p} \cdot \lambda^{*}$.

(iv) $\lambda \uparrow \mu$ if and only if $\tilde{\mu} \uparrow \tilde{\lambda}$.

(v) Both $\lambda$ and $\mu$ are in the closure of a facet if and only if both $\lambda^{*}$ and $\mu^{*}$ are in the closure of a facet.

By the above discussion we can see that $T_{\mu^{*}}^{\lambda^{*}}$ makes sense if $T_{\mu}^{\lambda}$ makes sense. If $M$ is a finite dimensional $G_{r} T$-module, so is the dual (as vector space) module $M^{*}$.

Proposition. Let $\mu, \lambda$ be weights in the closure of an alcove. For any finite dimensional $G_{r} T$-module $M,\left(T_{\lambda}^{\mu} M\right)^{*}=T_{\lambda^{*}}^{\mu^{*}}\left(M^{*}\right)$.

Proof. Recall that for a simple $G$-module $L(\nu)$ with $\nu$ dominant, we have $L(\nu)^{*}=L\left(\nu^{*}\right)$ and $L_{r}(\nu)^{*}=L_{r}(\bar{\nu})$ if $L_{r}(\nu)$ is a simple $G_{r} T$-module. Since all the modules in the category $\mathscr{U}$ of $G_{r} T$-modules are finite dimensional, taking 
dual defines an exact contravariant functor $*: \mathscr{U} \rightarrow \mathscr{U}$. It follows from the discussion above that the functor $*$ sends modules in the same block to modules in the same block. Therefore we get the following commutative diagram:

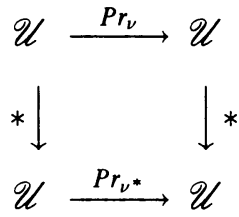

Thanks to the lemma above, we have $P r_{\nu^{*}}=P r_{\bar{\nu}}$. If $y \in W_{p}$ such that $\lambda=y \cdot \lambda_{C}$ and $\mu=y \cdot \mu_{C}$ with $\lambda_{C}, \mu_{C} \in \bar{C}$ (the closure of the bottom alcove), then $\lambda^{*}=y^{*} \cdot \lambda_{C}^{*}$ and $\mu^{*}=y^{*} \cdot \mu_{C}^{*}$ with $\lambda_{C}^{*}, \mu_{C}^{*} \in \bar{C}$. Now if $\nu=w(\mu-\lambda) \in X_{+}(T)$, then $\nu^{*}=w^{*}\left(\mu^{*}-\lambda^{*}\right) \in X_{+}(T)$ and $w^{*} \in W$. Now we have

$$
\left(T_{\lambda}^{\mu} M\right)^{*}=\operatorname{Pr}_{\mu^{*}}\left(L(\nu) \otimes P r_{\lambda} M\right)^{*}=\operatorname{Pr}_{\mu^{*}}\left(L(\nu)^{*} \otimes P r_{\lambda^{*}} M^{*}\right)=T_{\lambda^{*}}^{\mu^{*}} M^{*} .
$$

3.8. Recall from [13, II, Chapter 11] that $Z_{r}^{\prime}(\lambda)^{*}=Z_{r}^{\prime}(\tilde{\lambda})$ and $\operatorname{Cap} Z_{r}^{\prime}(\lambda)=$ $L_{r}(\tilde{\lambda})^{*}$. If $M$ is a finite dimensional module, then

(i) $\left(\operatorname{Soc}^{i} M\right)^{*}=\operatorname{Cap}^{i}\left(M^{*}\right)$.

(ii) $\operatorname{Cap}_{i} M=\left(\operatorname{Soc}_{i} M^{*}\right)^{*}$.

(iii) If $M$ is rigid, then $\operatorname{Cap}_{i} M=\operatorname{Soc}_{l l(M)-i+1} M$.

Now assume $\lambda$ and $\mu$ as in 3.4. We have studied the socle structure of the module $T_{\mu}^{\lambda} Z_{r}^{\prime}(\mu)$ in 3.5. In this subsection we study its radical structure. By Proposition 3.7 we have

$$
T_{\mu}^{\lambda} Z_{r}^{\prime}(\mu)=\left(T_{\mu^{*}}^{\lambda^{*}} Z_{r}^{\prime}(\tilde{\mu})\right)^{*} .
$$

Note that the map $w \cdot \lambda \mapsto \widetilde{w \cdot \lambda}$ defines a bijection between $W_{\mu} \cdot \lambda$ and $W_{\tilde{\mu}} \cdot \tilde{\lambda}$, which reverses the strong linkage order. Therefore $\tilde{\lambda}$ is the minimal element and $\tilde{\lambda}_{\#}$ is the maximal element in the set $W_{\tilde{\mu}} \cdot \tilde{\lambda}$. It follows from 3.5 that

$$
\left[\operatorname{Soc}^{l(\tilde{y})} T_{\mu^{*}}^{\lambda^{*}} Z_{r}^{\prime}(\tilde{\mu}): L_{r}(\tilde{y} \cdot \tilde{\lambda})\right]=0 .
$$

Now we can apply the duality functor to the above formula. Note that $L_{r}(\widetilde{y \cdot \lambda})^{*}$ is the simple cap of $Z_{r}^{\prime}(y \cdot \lambda)$.

Proposition. (i) $\left[\mathrm{Cap}^{l(y)} T_{\mu}^{\lambda} Z_{r}^{\prime}(\mu): L_{r}(\widetilde{y \cdot \lambda})^{*}\right]=0$ for all $y \in W_{\mu}$.

(ii) For all $m>0$ we have

$$
\left[\operatorname{Cap}^{m} T_{\mu}^{\lambda} Z_{r}^{\prime}(\mu)\right] \leq \sum_{w \in W_{\mu}} \sum_{j}\left[\operatorname{Cap}_{j} Z_{r}^{\prime}(w \cdot \lambda): L_{r}(\tilde{\lambda})^{*}\right]\left[\operatorname{Cap}^{m+1-j} Z_{r}^{\prime}(w \cdot \lambda)\right] .
$$

Proof. (i) follows from the above argument. Similarly, by using (5), we can apply the duality functor to the formula in 2.4 for the module $T_{\mu^{*}}^{\lambda^{*}} Z_{r}^{\prime}(\tilde{\mu})$ to get (ii).

\section{RIGIDITY}

4.1. Motivated by Irving's work on the structure of Verma modules, Andersen and Kaneda [2] proved that, for a $p$-regular weight $\lambda$, the injective module $Q_{1}(\lambda)$ and the induced module $Z_{1}^{\prime}(\lambda)$ are rigid if the Lusztig conjecture is true. 
Furthermore they proved that the socle filtration of $Z_{1}^{\prime}(\lambda)$ can be calculated from the generic inverse Kazhdan-Lusztig polynomials and the formula given in 2.4 for the injective module $Q_{1}(\lambda)$ is an equality. What they used in their proof is the conjecture formulated by Andersen [1] analogous to the Vogan conjecture for semisimple Lie algebras (sometimes called the Vogan conjecture also), which is equivalent to the Lusztig conjecture. Let $\lambda$ be a $p$-regular weight and $\mu$ a weight in the closure of the alcove containing $\lambda$ such that $\left|W_{\mu}\right|=2$. Now the Vogan conjecture can be stated as follows.

Conjecture. Let $L$ be a simple module in $\mathscr{U}_{\mu}$. Then $l l\left(T_{\mu}^{\lambda} L\right) \leq 3$.

The following facts have been proved by Andersen and Kaneda in [2] assuming the Vogan conjecture, when $\lambda$ is a $p$-regular weight.

(i) $l l\left(Q_{1}(\lambda)\right)=1+2 N$.

(ii) $l l\left(Z_{1}^{\prime}(\lambda)\right)=1+N$.

(iii) Both $Q_{1}(\lambda)$ and $Z_{1}^{\prime}(\lambda)$ are rigid.

Here $N=l\left(w_{0}\right)$. Throughout this section we assume the Vogan conjecture and $r=1$.

4.2. Theorem. Let $\lambda$ be p-regular and $\mu$ a weight in the closure of the alcove containing $\lambda$. Then

(i) $l l\left(Z_{1}^{\prime}(\mu)\right)=N+1-l\left(w_{\mu}\right)$.

(ii) $l l\left(Q_{1}(\mu)\right)=2 N+1-2 l\left(w_{\mu}\right)$.

(iii) $l l\left(T_{\mu}^{\lambda} Z_{1}^{\prime}(\mu)\right)=N+1+l\left(w_{\mu}\right)$.

Proof. Recall that Andersen and Kaneda have shown in [2] by considering intertwining maps that $l l\left(Z_{1}^{\prime}(\mu)\right) \geq N+1-l\left(w_{\mu}\right)$. Now (i) is a consequence of 3.4(iii). Let $\lambda$ be maximal in the set $W_{\mu} \cdot \lambda$. Then we have $T_{\mu}^{\lambda} Q_{1}(\mu)=Q_{1}\left(\lambda_{\#}\right)$. Now it follows from 4.1(i) and 3.6(ii) that $l l\left(Q_{1}(\mu)\right) \leq 2 N+1-2 l\left(w_{\mu}\right)$. Noting that $W_{\mu} \cong W_{w_{0}\left(\mu_{0}\right)+p \mu_{1}+2(p-1) \rho}$, the equality in (ii) is a consequence of (i) and Corollary (B) in 2.7 .

To prove (iii) we consider the injectives in the category $\mathscr{U}_{t}(\lambda)$. We use induction on $l(w)$ to show that $l l(I(w \cdot \lambda)) \leq l l\left(Z_{1}^{\prime}(\lambda)\right)+l(w)$ for $w \in W_{\mu}$. If $l(w)=0$, we have $I(\lambda)=Z_{1}^{\prime}(\lambda)$. In general, let $w \in W_{\mu}$. Suppose we are done for all $y \in W_{\mu}$ with $l(y)<l(w)$. Let $s \in W_{\mu}$ be a reflection in a wall of the alcove containing $w \cdot \lambda$ such that $s w \cdot \lambda>w \cdot \lambda$. Therefore, $l(s w)=l(w)-1$. Consider the translation functor $\theta_{s}$ across the $s$-wall. Since $\theta_{s}$ annihilates the socle $L_{1}(s w \cdot \lambda)$ of $I(s w \cdot \lambda)$, we can use the generalized Vogan conjecture [2, Theorem 5.2] to get

$$
l l\left(\theta_{s} I(s w \cdot \lambda)\right)=l l\left(\theta_{s}\left(I(s w \cdot \lambda) / L_{1}(s w \cdot \lambda)\right)\right) \leq l l(I(s w \cdot \lambda))+1 .
$$

If we can show $L_{1}(w \cdot \lambda) \subseteq \theta_{s} I(s w \cdot \lambda), I(w \cdot \lambda)$ will be a summand of $\theta_{s} I(s w \cdot \lambda)$ since $\theta_{s}$ sends injective modules in $\mathscr{U}_{t}(\lambda)$ to injective modules in $\mathscr{U}_{t}(\lambda)$ and we are done. In fact, we have

$$
\begin{aligned}
& \operatorname{Hom}_{\mathscr{U}_{t}(\lambda)}\left(L_{1}(w \cdot \lambda), \theta_{s} I(s w \cdot \lambda)\right) \\
& \quad=\operatorname{Hom}_{\mathscr{U}_{t}(\lambda)}\left(\theta_{s} L_{1}(w \cdot \lambda), I(s w \cdot \lambda)\right)=\left[\theta_{s} L_{1}(w \cdot \lambda): L_{1}(s w \cdot \lambda)\right]=1
\end{aligned}
$$

since $I(s w \cdot \lambda)$ is the injective hull of $L_{1}(s w \cdot \lambda)$.

Now if we take $w=w_{\mu}$ we get $l l\left(T_{\mu}^{\lambda} Z_{1}^{\prime}(\mu)\right) \leq N+1+l\left(w_{\mu}\right)$. The other direction is a consequence of (i) and 3.6(ii). 
4.3. Now we can use the results of 4.2 to refine 3.4 as follows. Suppose one has $\left[\operatorname{Soc}^{l(w)+1} Z_{1}^{\prime}\left(w \cdot \lambda_{\#}\right): L_{1}\left(\lambda_{\#}\right)\right]=0$. Then

$$
\operatorname{Soc}^{l(w)+1} Z_{1}^{\prime}\left(w \cdot \lambda_{\#}\right) \subseteq \operatorname{Ker}\left(Z_{1}^{\prime}\left(w \cdot \lambda_{\#}\right) \rightarrow Z_{1}^{\prime}\left(\lambda_{\#}\right)\right)
$$

and thus $T_{\mu}^{\lambda} \operatorname{Soc}^{l(w)+1} Z_{1}^{\prime}\left(w \cdot \lambda_{\#}\right)=0$.

On the other hand, we consider the composite $\phi$ of the maps constructed in 3.4 such that the map $\phi: Z_{1}^{\prime}(\lambda) \rightarrow Z_{1}^{\prime}\left(w \cdot \lambda_{\#}\right)$ annihilates $\operatorname{Soc}^{l\left(w w_{\mu}\right)} Z_{1}^{\prime}(\lambda)$. Thus we have

$$
\phi\left(Z_{1}^{\prime}(\lambda)\right) \subseteq \operatorname{Soc}^{l l\left(Z_{1}^{\prime}(\lambda)\right)-l\left(w w_{\mu}\right)} Z_{1}^{\prime}\left(w \cdot \lambda_{\#}\right) .
$$

This yields $T_{\lambda}^{\mu}\left(\operatorname{Soc}^{l l\left(Z_{1}^{\prime}(\lambda)\right)-l\left(w w_{\mu}\right)} Z_{1}^{\prime}\left(w \cdot \lambda_{\#}\right)\right)=Z_{1}^{\prime}(\mu)$. So combining the above argument we get $l l\left(Z_{1}^{\prime}(\mu)\right) \leq l l\left(Z_{1}^{\prime}(\lambda)\right)-l\left(w w_{\mu}\right)-l(w)-1=l l\left(Z_{1}^{\prime}(\lambda)\right)-$ $l\left(w_{\mu}\right)-1$. This contradicts the Loewy length we got in 4.2 . Therefore we have the following for $w \in W_{\mu}$ :

$$
\left[\operatorname{Soc}_{j} Z_{1}^{\prime}\left(w \cdot \lambda_{\#}\right): L_{1}\left(\lambda_{\#}\right)\right]= \begin{cases}1 & \text { if } j=l(w)+1, \\ 0 & \text { otherwise. }\end{cases}
$$

Remark. Actually, the above result does not need Vogan's conjecture. For one can use the result of Andersen and Kaneda as mentioned in the proof of 4.2(i), which is true without Vogan conjecture.

4.4. Now we can prove our main result on translation functors.

Theorem. Let $\lambda$ be a p-regular weight and $\mu$ a weight in the closure of the alcove containing $\lambda$. If the Vogan conjecture is true, then $T_{\mu}^{\lambda} Z_{1}^{\prime}(\mu)$ is rigid and for any $m>0$,

$$
\left[\operatorname{Soc}^{m} T_{\mu}^{\lambda} Z_{1}^{\prime}(\mu)\right]=\sum_{w \in W_{\mu}}\left[\operatorname{Soc}^{m-l(w)} Z_{1}^{\prime}\left(w \cdot \lambda_{\#}\right)\right] .
$$

Here $\lambda_{\#}$ is the minimal element in $W_{\mu} \cdot \lambda$.

Proof. Keeping in mind that $Z_{1}^{\prime}\left(w \cdot \lambda_{\#}\right)$ is rigid for all $w \in W_{\mu}$, we can use Proposition 2.4, Proposition 3.8, and (6) to get the following calculation for $m>0$.

$$
\begin{aligned}
{\left[\operatorname{Soc}^{m}\right.} & \left.T_{\mu}^{\lambda} Z_{1}^{\prime}(\mu)\right]+\left[\operatorname{Cap}^{N+1+l\left(w_{\mu}\right)-m} T_{\mu}^{\lambda} Z_{1}^{\prime}(\mu)\right] \\
& \leq \sum_{w \in W_{\mu}}\left(\left[\operatorname{Soc}^{m-l(w)} Z_{1}^{\prime}\left(w \cdot \lambda_{\#}\right)\right]+\left[\operatorname{Cap}^{N+1+l\left(w_{\mu}\right)-m-l\left(w w_{\mu}\right)} Z_{1}^{\prime}\left(w \cdot \lambda_{\#}\right)\right]\right) \\
& =\sum_{w \in W_{\mu}}\left(\left[\operatorname{Soc}^{m-l(w)} Z_{1}^{\prime}\left(w \cdot \lambda_{\#}\right)\right]+\left[\operatorname{Cap}^{N+1-(m-l(w))} Z_{1}^{\prime}\left(w \cdot \lambda_{\#}\right)\right]\right) \\
& =\sum_{w \in W_{\mu}}\left[Z_{1}^{\prime}\left(w \cdot \lambda_{\#}\right)\right]=\left[T_{\mu}^{\lambda} Z_{1}^{\prime}(\mu)\right] .
\end{aligned}
$$

This shows that $T_{\mu}^{\lambda} Z_{1}^{\prime}(\mu)$ is rigid and the inequalities become equalities.

4.5. By Theorem 4.2, we have $l l\left(Q_{1}(\mu)\right)=2 l l\left(Z_{1}^{\prime}(\mu)\right)-1$. Note that the Loewy length of $Z_{1}^{\prime}(\mu)$ depends only on the $W_{p}$-orbit of $\mu$, i.e., $l l\left(Z_{1}^{\prime}(\mu)\right)=l l\left(Z_{1}^{\prime}\left(\mu^{\prime}\right)\right)$ if $\mu^{\prime} \in W_{p} \cdot \mu$.

On the other hand, we can consider the dual module $Q_{1}(\mu)^{*}=Q_{1}(\bar{\mu})$ which is the injective hull of $L_{1}(\mu)^{*}=L_{1}(\bar{\mu})$ in $\mathscr{U}_{\mu}$. Note that taking duals induces 
an automorphism of the Grothendieck group of the category $\mathscr{U}_{\mu}$. So from the argument of 3.8 on taking duals and the formula of 2.4 (for the category $\mathscr{U}_{\mu}$ ) we can get the following ( $r$ is not necessarily 1$)$ :

$$
\begin{aligned}
{\left[\operatorname{Cap}^{n} Q_{r}(\mu)\right] } & =\left[\operatorname{Soc}^{n} Q_{r}(\bar{\mu})\right]^{*} \\
& \leq \sum_{\nu} \sum_{j}\left[\operatorname{Cap}_{j} Z_{r}^{\prime}(\nu): L_{r}(\mu)\right]\left[\operatorname{Cap}^{n+1-i} Z_{r}^{\prime}(\nu)\right] .
\end{aligned}
$$

Lemma. If $Z_{1}^{\prime}(\nu)$ is rigid for all $\nu$ such that $\left[Z_{1}^{\prime}(\mu): L_{1}(\nu)\right] \neq 0$, so is $Q_{1}(\mu)$ and

$$
\left[\operatorname{Soc}^{m} Q_{1}(\mu)\right]=\sum_{\nu} \sum_{j}\left[\operatorname{Soc}_{j} Z_{1}^{\prime}(\nu): L_{1}(\mu)\right]\left[\operatorname{Soc}^{m+1-j} Z_{1}^{\prime}(\nu)\right] .
$$

Proof. Note the rigidity of $Z_{1}^{\prime}(\nu)$ gives $\operatorname{Cap}_{i} Z_{1}^{\prime}(\nu)=\operatorname{Soc}_{l l\left(Z_{1}^{\prime}(\nu)\right)-i+1} Z_{1}^{\prime}(\nu)$. Now we can use (8) for $Q_{1}(\mu)$ to calculate for $n>0$ the following:

$$
\begin{aligned}
& {\left[\operatorname{Soc}^{n} Q_{1}(\mu)\right]+\left[\operatorname{Cap}^{2 l l\left(Z_{1}^{\prime}(\mu)\right)-1-n} Q_{1}(\mu)\right]} \\
& \leq \sum_{\nu}\left(\sum_{i}\left[\operatorname{Soc}_{i} Z_{1}^{\prime}(\nu): L_{1}(\mu)\right]\left[\operatorname{Soc}^{n+1-i} Z_{1}^{\prime}(\nu)\right]\right. \\
& \left.\quad+\sum_{j}\left[\operatorname{Cap}_{j} Z_{1}^{\prime}(\nu)^{\prime}: L_{1}(\mu)\right]\left[\operatorname{Cap}^{2 l l\left(Z_{1}^{\prime}(\mu)\right)-n-j} Z_{1}^{\prime}(\nu)\right]\right) \\
& =\sum_{\nu} \sum_{i}\left[\operatorname{Soc}_{i} Z_{1}^{\prime}(\nu): L_{1}(\mu)\right] \\
& \quad \cdot\left(\left[\operatorname{Soc}^{n+1-i} Z_{1}^{\prime}(\nu)\right]+\left[\operatorname{Cap}^{2 l l\left(Z_{1}^{\prime}(\mu)\right)-l l\left(Z_{1}(\nu)\right)-n+i-1} Z_{1}^{\prime}(\nu)\right]\right) \\
& \left.=\sum_{\nu} \sum_{i}\left[\operatorname{Soc}_{i} Z_{1}^{\prime}(\nu): L_{1}(\mu)\right]\left[Z_{1}^{\prime}(\nu)\right]=\left[Q_{1}(\mu)\right)\right] .
\end{aligned}
$$

Here the equalities follow from rigidity of $Z_{1}(\nu)$ and $l l\left(Z_{1}^{\prime}(\nu)\right)=l l\left(Z_{1}^{\prime}(\mu)\right)$. So the lemma follows.

Remark. If $\mu$ is $p$-regular, this result has been proved by Andersen and Kaneda by using the duality functor $D$ and the formulation of the socle series of $Z_{1}^{\prime}(\nu)$ in terms of $Q$-polynomials with some manipulation of the $Q$-polynomials.

4.6. Let us return to the category $\mathscr{U}_{t}(\lambda)$ with $r=1$ and $p$-regular $\lambda$. Recall that $L_{1}(\overline{\tilde{\lambda}})=\operatorname{Cap} Z_{1}^{\prime}(\lambda)$. As we have seen in the proof of Corollary (B) in 2.7 that $Q_{1}(\overline{\tilde{\lambda}})$ is in the category $\mathscr{U}_{t}(\lambda)$, we have $I(\overline{\tilde{\lambda}})=Q_{1}(\overline{\tilde{\lambda}})$.

Theorem. Let $r=1$ and $\lambda$ be p-regular. Suppose the Vogan conjecture is true. If $\nu \uparrow \lambda$ and $I(\nu)$ is the injective hull of $L_{1}(\nu)$ in $\mathscr{U}_{t}(\lambda)$, then

$$
\left[\operatorname{Soc}^{m} I(\nu)\right]=\sum_{\mu \uparrow \lambda} \sum_{j}\left[\operatorname{Soc}_{j} Z_{r}^{\prime}(\mu): L_{r}(\nu)\right]\left[\operatorname{Soc}^{m+1-j} Z_{r}^{\prime}(\mu)\right] .
$$

Thus the inequality in 2.4 becomes equality.

Proof. Here the proof is similar to Irving's proof for the socle filtration of the projective modules in the category $\mathscr{O}$ in [10]. The idea is to show that all the socles and caps of the factors $Z_{1}^{\prime}(\mu)$ in a good filtration of $I(\nu)$ are in the socle layer as described by the formula. Then the rigidity of $Z_{1}^{\prime}(\mu)$ will force all of their socle layers to be placed in the socle layer of $I(\mu)$ as described by the 
formula. However the socles of all the factors $Z_{1}^{\prime}(\mu)$ are in the desired socle layers of $I(\nu)$ by 2.6. So what we need to do is to show

$$
\left[\operatorname{Soc}_{m} I(\nu): L_{1}(\tilde{\mu})^{*}\right]=\sum_{\eta \uparrow \lambda} \sum_{i}\left[\operatorname{Soc}_{i} Z_{1}^{\prime}(\eta): L_{1}(\nu)\right]\left[\operatorname{Soc}_{m+1-i} Z_{1}^{\prime}(\eta): L_{1}(\tilde{\mu})^{*}\right] .
$$

It follows from Theorem 2.5 that $\left[\operatorname{Soc}_{m} I(\nu): L_{1}(\tilde{\mu})^{*}\right]=\left[\operatorname{Soc}_{m} I(\tilde{\mu}): L_{1}(\nu)\right]$. Since $\mu \uparrow \lambda$, we have $I(\tilde{\tilde{\mu}})=Q_{1}(\overline{\tilde{\mu}})$. Now we have proved in 4.5 the following:

$$
\begin{aligned}
{\left[\operatorname{Soc}_{m} Q_{1}(\tilde{\mu}): L_{1}(\nu)\right] } & =\sum_{\eta} \sum_{i}\left[\operatorname{Soc}_{i} Z_{1}^{\prime}(\eta): L_{1}(\tilde{\mu})^{*}\right]\left[\operatorname{Soc}_{m+1-i} Z_{1}^{\prime}(\eta): L_{1}(\nu)\right] \\
& =\sum_{\eta} \sum_{i}\left[\operatorname{Soc}_{i} Z_{1}^{\prime}(\eta): L_{1}(\nu)\right]\left[\operatorname{Soc}_{m-i+1} Z_{1}^{\prime}(\eta): L_{1}(\tilde{\mu})^{*}\right]
\end{aligned}
$$

Note that whenever $\left[Z_{1}^{\prime}(\eta): L_{1}(\tilde{\mu})^{*}\right] \neq 0, Z_{1}^{\prime}(\eta)$ is a factor of $Q_{1}(\overline{\tilde{\mu}})$ and $\eta \uparrow \mu \uparrow \lambda$. So we get (10) and the theorem follows.

\section{ACKNOWLEDGMENT}

I am grateful to Ron Irving for giving me the preprints of his research papers and discussing the ideas in his papers with me, and to Jens Jantzen for discussing some of the facts in this paper and referring me to Donkin's work. I would also like to thank John Sullivan and David Collingwood for their hospitality during my stay at the University of Washington.

\section{REFERENCES}

1. H. H. Andersen, An inversion formula for the Kazhdan-Lusztig polynomials for affine Weyl groups, Adv. in Math. 60 (1986), 125-153.

2. H. H. Andersen and M. Kaneda, Loewy series of modules for the first Frobenius kernel in a reductive algebraic group, Proc. London Math. Soc. (3) 59 (1989), 74-98.

3. E. Cline, B. Parshall, and L. Scott, Algebraic stratification in representation categories, J. Algebra 117 (1988), 504-521.

4. _ Duality in highest weight categories, Classical Groups and Related Topics, Contemp. Math., vol. 82, Amer. Math. Soc., Providence, R. I., 1989, pp. 7-22.

5. S. Donkin, Rational representations of algebraic groups, Lecture Notes in Math., vol. 1140, Springer-Verlag, Berlin and New York, 1985.

6. __ Skew modules for reductive groups, J. Algebra 113 (1988), 465-479.

7. S. R. Doty, Character formulas and Frobenius subgroups of algebraic groups, J. Algebra 125 (1989), 331-347.

8. T. J. Enright and B. Shelton, Categories of highest weight modules: applications to classical Hermitian symmetric pairs, Mem. Amer. Math. Soc., vol. 67, no. 367, 1987.

9. R. S. Irving, Projective modules in the category $\mathscr{O}_{S}:$ Loewy series, Trans. Amer. Math. Soc. 291 (1985), 733-754.

10. __ The socle filtration of a Verma module, Ann. Sci. École Norm. Sup. 21 (1988), 47-65.

11. __ Loewy filtrations of Weyl modules, Trans. Amer. Math. Soc. (to appear).

12. __ Singular blocks of the category O) , Math. Z. 204 (1990), 209-224.

13. J. C. Jantzen, Representations of algebraic groups, Academic Press, Orlando, 1987.

14. M. Kaneda, Extensions of modules for infinitesimal algebraic groups, J. Algebra 122 (1989), 188-210.

15. M. Koppinen, On the translation functors for a semisimple algebraic group, Math. Scand. 51 (1982), 217-226. 
16. __ Homomorphisms between neighboring Weyl modules, J. Algebra 103 (1984), 302-319.

17. Z. Lin, The structure of cohomology of line bundles on the flag varieties for some groups of rank 2, Ph. D. Thesis, Univ. of Massachusetts, 1989.

18. _ Extensions between simple modules for Frobenius kernels, J. Pure Appl. Algebra 72 (1991), 275-294.

19. H. Schubert, Categories, Springer-Verlag, New York and Berlin, 1972.

Department of Mathematics, University of Washington, Seattle, Washington 98195 